\title{
Novel Faraday Rotator by Use of Cryogenic TGG Ceramics
}

\author{
Ryo YASUHARA, ${ }^{1,3}$ Shigeki TOKITA, ${ }^{2}$ Junji KAWANAKA, ${ }^{3}$ Hirofumi KAN, ${ }^{1}$ \\ Toshiyuki KAWASHIMA, ${ }^{1}$ Hideki YAGI, ${ }^{4}$ Hoshiteru NOZAWA, ${ }^{4}$ Takagimi YANAGITANI, ${ }^{4}$ \\ Yasushi FUJIMOTO, ${ }^{3}$ Hidetsugu YOSHIDA, ${ }^{3}$ and Masahiro NAKATSUKA ${ }^{3}$ \\ ${ }^{1}$ Hamamatsu Photonics K. K., 5000, Hirakuchi, Hamakita-ku, Hamamatsu, Shizuoka 434-8601 \\ ${ }^{2}$ Institute for Chemical Research, Kyoto University, Gokasyo, Uji, Kyoto 611-0011 \\ ${ }^{3}$ Institute of Laser Engineering, Osaka University, 2-6, Yamada-oka, Suita, Osaka 565-0871 \\ ${ }^{4}$ Material Development Dept., Konoshima Chemical Co. Ltd., 80 Koda, Takuma, Mitoyo, Kagawa 769-1103
}

(Received January 18, 2008)

\begin{abstract}
We have proposed the cryogenic TGG ceramics based Faraday rotator for High peak and high average power laser. As the first demonstration of Faraday effect in a TGG ceramics, its Verdet constant at $1053 \mathrm{~nm}$ is evaluated to be $36.4 \mathrm{rad} / \mathrm{Tm}$ at room temperature which is same as that of the single crystal. In addition, the temperature dependence of Verdet constant is obtained experimentally. At liquid helium temperature, it is 87 times greater than that at room temperature. The measured thermal conductivity was corresponding to TGG single crystal.
\end{abstract}

Key Words: Magnet-optical material, Faraday effect, High average power laser

\section{Introduction}

Recently, pulse lasers with both high pulse energy and high average power are strongly desired for various industrial and scientific applications such as laser peening, ${ }^{1)}$ laser processing, ${ }^{2,}{ }^{3)}$ high-energy particle generation, ${ }^{4)}$ hard $\mathrm{x}$-ray generation, ${ }^{5)}$ inertial fusion energy (IFE ${ }^{6-8)}$ and so on. A Faraday element is one of the indispensable key optics for isolation of laser chains and birefringence compensation of laser materials in such high-power laser systems. There are three critical factors of high Verdet constant, high thermal strength and size scalability for the Faraday elements. Terbium-doped materials are the most popular due to a high Verdet constant. A terbium-doped glass has been often used for high pulse energy lasers due to its superior size scalability. ${ }^{6)}$ Its low thermal characteristics (thermal conductivity of Tb:doped glass FR-5 is $0.84 \mathrm{~W} / \mathrm{mK}){ }^{7,9)}$ however, is not preferred for repeatable operation. On the other hand, a Faraday crystal shows excellent thermal conductivity to be suitable for high average power operation, but it is difficult to obtain a large aperture crystal. Many researches for development of Faraday media were reported for the fiber laser, ${ }^{10,11)}$ optical wave guide $^{12)}$ and solid-state laser system, ${ }^{13,14)}$ however those for high pulse energy and high average power operation has not been reported yet.

As one of solutions for the realization, a terbium gallium garnet (TGG) ceramics will be a promising Faraday element due to its high Verdet constant of 36-40 rad/Tm, ${ }^{15-17)}$ high thermal conductivity of 4.5-7.4 W/mK, ${ }^{16-22)}$ and excellent size scalability. The TGG ceramics were reported in 2003 for the first time. ${ }^{23)}$ Then, the theoretical analysis of thermal birefringence in TGG ceramics was reported. ${ }^{24)}$ However, no Faraday effect measurement with TGG ceramics has been demonstrated because of its low optical quality. The recent ceramics technology ${ }^{25-27)}$ enables us to produce laser-graded TGG ceramics. The size is still small now (Our sample size is
$5.95 \mathrm{~mm}$ in length and $5 \mathrm{~mm} \times 1 \mathrm{~mm}$ cross section). The large aperture ceramics will be obtained by optimizing the process condition in future.

In an extremely high pulse energy laser system such as IFE lasers, there is another problem of a strong magnetic field, which is difficult to be isolated. The strong magnetic field often interferes with electronic control systems and diagnostic devices. Also, a huge magnet system is necessary. In fact, a huge superconductive solenoid has been used in fusion lasers. ${ }^{6)}$ By improving a Verdet constant, the required magnetic field strength will reduce and, the whole magnetic system size will become compact. Cryogenic cooling of materials often becomes an easy and effective method to control various material coefficients. Recently, cryogenic cooling of several active elements concerning to solid-state lasers has been demonstrated to improve thermal characteristics and to tune a stimulated emission cross section. ${ }^{28-32)}$ Using TGG crystal, some groups were estimated the Verdet constant at temperatures in the range between $77 \mathrm{~K}$ and $300 \mathrm{~K}$ by measuring the rotation angle at a constant magnetic field. ${ }^{33-34)}$

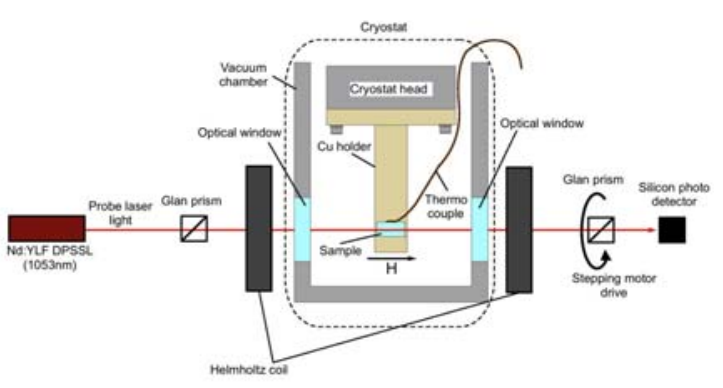

Fig. 1. A schematic diagram of the experimental setup for the measurement of the Verdet constant. 
At $77 \mathrm{~K}$, the Faraday rotation angle was 3.5 times as large as that at $293 \mathrm{~K}$.

In this paper, we have measured the temperature dependence of the Verdet constant of laser-graded TGG ceramics at 1053 $\mathrm{nm}$ wavelength. Also, a TGG single crystal was used for comparison. The Verdet constant difference between the ceramics and the crystal is only $0.8 \%$. The Verdet constant of TGG ceramics increases to $1453 \mathrm{rad} / \mathrm{Tm}$ at $7.8 \mathrm{~K}$, which is 40 times higher than that at $300 \mathrm{~K}$. This will be one of the promising Faraday materials for high-power lasers for IFE. The measured thermal conductivity was corresponding to TGG single crystal. This will be one of promising Faraday materials for high-peak and high average power lasers.

\section{Experimental Setup}

\subsection{Verdet constant}

Experimental procedures for the Verdet constant measurements of TGG ceramics are as follows. The laser light passes through a sample, which is between a pair of Glan laser prisms used as an analyzer and polarizer, respectively. The transmitted laser intensity is measured by a silicon photo diode. A polarization plane of laser light is rotated due to the Faraday effect induced by a magnetic field. The transmitted laser intensity I is expressed by the Malus's law. A diode-pumped Nd:YLF continuous wave laser (IRCL-100-1053, CrystaLaser) is used as an optical source. The wavelength and maximum output power are $1053 \mathrm{~nm}$ and $500 \mathrm{~mW}$, respectively. The extinction ratio of the prism is $0.2 \times 10^{5}$. The analyzer prism is attached to a stepping-motor-driven rotation stage, whose surface plane is perpendicular to the optical axis. A TGG ceramic (Konoshima Chemical Co., Ltd., $5.95 \mathrm{~mm}$ in length and $5 \mathrm{~mm} \times 1 \mathrm{~mm}$ cross section) and a TGG single crystal (OFR, Inc) with $<111>$ orientation $(12.75 \mathrm{~mm}$ in length and $4.7 \mathrm{~mm}$ in diameter) are used as Faraday materials. Each of them is cramped by a copper holder. A thin indium hoil is used to improve the thermal contact between the material and the holder. The holder is attached to a temperature-controllable cryostat (Iwatani HE05) and set in a vacuum chamber. The temperature is measured by a calibrated $\mathrm{Kp}$-Au thermo couple on the material surface. The controllable temperature range is between $7.8 \pm 2.1$ and $300 \pm 0.1 \mathrm{~K}$. A spatially uniform magnetic field is applied to the material by using double Helmholtz coils. The applied magnetic field is temporally pulsed with duration of $100 \mathrm{~ms}$ in order to suppress the coil heating. The magnitude of the magnetic field is $253 \mathrm{G}$ at maximum. The magnetic field strength, which is calibrated with a Gauss meter (MODEL5080, F.W. Bell), is controlled by adjusting an electric current of the Helmholtz coils. The laser intensity $I$ is measured at the uniform time-region of top-hat temporal magnetic field, then the fluctuation of the detected intensity $I$ is $0.8 \%$ RMS. The extinction ratio of the transmitted laser intensity is maintained better than 1:5000 during measurements. In consequence the accuracy of the rotation angle of this system is estimated to be better than $17.44 \mathrm{mrad}$.

\subsection{Thermal conductivity}

The thermal conductivity of TGG ceramics is measured by the steady-state longitudinal heat flow method. The sample size of the TGG ceramics is $5.95 \mathrm{~mm}$ in length and $5 \mathrm{~mm} \times 1$ $\mathrm{mm}$ cross section. The ceramics are made by Konoshima Chemical Co., Ltd. A face of the TGG ceramics is thermally contacted with the cooling head of the cryostat. And we attach

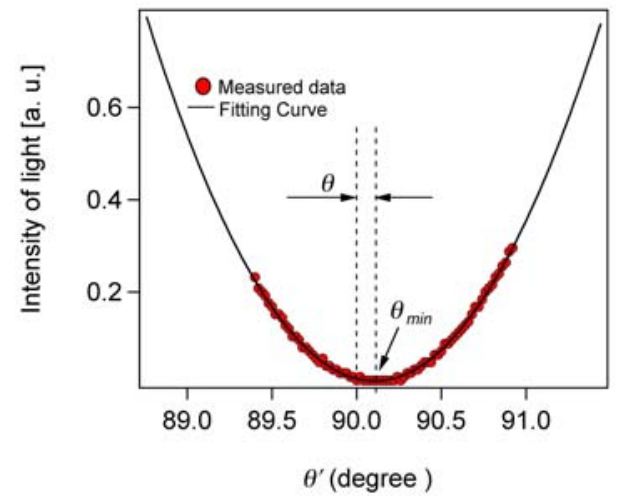

Fig. 2. Curve fitting of the light intensity and rotation angle of the TGG ceramics at $300 \mathrm{~K}$.

the heater at the other side of the TGG ceramics. The temperatures at the two different positions of the TGG ceramics were measured by thermocouples. The equation of $\kappa=\mathrm{QS} / \Delta \mathrm{TLt}$ is used for determination of the thermal conductivity. $\mathrm{Lt}(\mathrm{m})$ is the distance between the thermocouples. $\mathrm{Q}(\mathrm{W})$ is the heating power of the heater. $\mathrm{S}\left(\mathrm{m}^{2}\right)$ is a cross section of the TGG ceramics. $\Delta \mathrm{T}(\mathrm{K})$ is the difference between the temperatures measured by the thermocouples.

\section{Result and discussion}

Figure 2 shows the transmitted laser intensity as a function of $\theta$. Solid line shows a fitting curve from a theoretical model.

${ }^{35)}$ Then, we can determine the Faraday rotation angle $\theta$ as a difference between $\pi / 2$ and $\theta^{\prime}=\theta_{\min }$, where $I$ is minimum. Using the measured angle $\theta$, the Verdet constant is obtained from the relation $\theta=V H L$ with the measured $\mathrm{H}$ and $\mathrm{L}$. At $300 \mathrm{~K} \pm 0.1 \mathrm{~K}$, the measured Verdet constant of the TGG single crystal is $36.2 \mathrm{rad} / \mathrm{Tm}$. That agreed with the published values $(35-40 \mathrm{rad} / \mathrm{Tm}){ }^{14-16)}$ The Verdet constant of the TGG ceramics is $36.4 \mathrm{rad} / \mathrm{Tm}$ at $300 \mathrm{~K} \pm 0.1 \mathrm{~K}$ is almost same as that of the single crystal, and the tiny difference is within the experimental error. The TGG ceramic is composed of TGG single crystal grains and boundary layers. When the boundary layer is very thin, it has no differences in quality between the ceramic grain and the single crystal. If the larger-size ceramics are obtainable, the higher pulse energy operation will be realized.

The measured Verdet constants V of the TGG single crystal and TGG ceramic are plotted in Fig. 3 as a function of the absolute temperature. The Verdet constant of the ceramic at $7.8 \mathrm{~K}$ is $1453 \mathrm{rad} / \mathrm{Tm}$, which is 40 times as large as that at $300 \mathrm{~K}$. In addition, $1122 \mathrm{rad} / \mathrm{Tm}$ is obtained for the single crystal at $9.1 \mathrm{~K}$. Within this temperature range, the experimental data are inversely proportional to the absolute temperature and fitted well to the theoretical model. The slope of the straight line is

$$
\frac{\operatorname{ANJ}(J+1) g^{2} \mu_{B}^{2}}{3 k}=13290 \pm 171.4 \mathrm{radK} / \mathrm{Tm}
$$

In consequence, the Verdet constant at $4.2 \mathrm{~K}$ is extrapolated at 


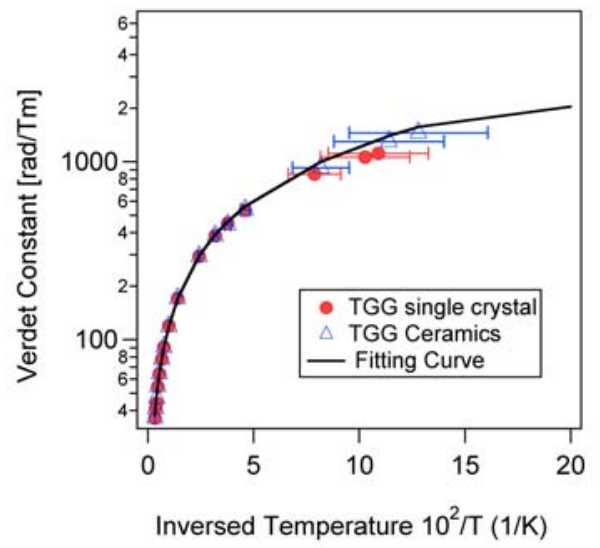

Fig. 3. The temperature dependence of Verdet constant of TGG single crystal and TGG ceramics.

$3164 \mathrm{rad} / \mathrm{Tm}$ from Eq. (1). Therefore, the required magnetic field is reduced to $1 / 87$, resulting in the stable electric control of the laser system, the accurate diagnostics and the reduced magnetic system size. In addition, the length of the Faraday material can be shortened to 1/87 in the same magnetic field, which gives an advantage of low spectral dispersion for short pulse lasers in the femtosecond regime.

Our measured thermal conductivity of the ceramics, as shown in Fig. 4, is $4.94 \pm 0.25 \mathrm{~W} / \mathrm{mK}$ that agrees with the published values $(4.5 \mathrm{~W} / \mathrm{mK}-7.4 \mathrm{~W} / \mathrm{mK}){ }^{20-23)}$ And the temperature behaviors of the thermal conductivity for both the single crystals and the ceramics are similar above about $100 \mathrm{~K}$. From $100 \mathrm{~K}$ to $50 \mathrm{~K}$, the influence of the Umklapp scattering decreases, and the thermal conductivity is limited by the grain boundary scattering in the ceramics. Compared with terbium-doped glass materials at room temperature, the magnitude is about one order high at the temperature between $100 \mathrm{~K}$ and $300 \mathrm{~K}$, which is preferable for high average power operation.

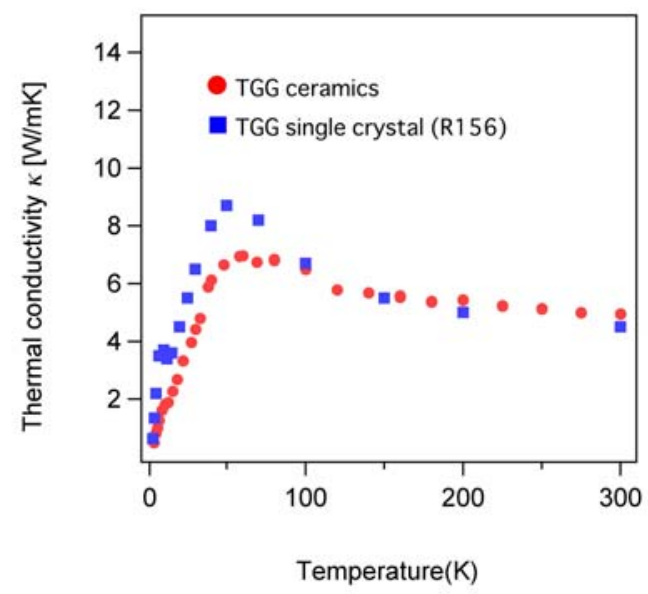

Fig. 4. The temperature dependence of thermal conductivity of TGG single crystal ${ }^{22)}$ and TGG ceramics.

\section{Conclusion}

The temperature dependence of the Verdet constant and thermal conductivity of the paramagnetic TGG ceramics has been measured for the first time to our knowledge. The temperature dependence of its magnitude is almost the same as that of a single crystal. If the larger-size ceramics are obtainable, the higher pulse energy operation will be realized. As the temperature decreases, the Verdet constant increases. For example, the magnitude at liquid helium temperature is 87 times as large as that at room temperature. The required magnetic field can be reduced, resulting in the stable electric control system without electromagnetic interference, the accurate diagnostics and the reduced magnetic system size. Also, the shortened Faraday material with a large cross sectioning area may be used in femtosecond lasers and in high average power lasers with an advantage of lower spectral dispersion and with the high thermal conductivity of sapphire sandwiched thin disk faraday medium. In addition to these improvements, the observed thermal conductivity of ceramics is one order higher than glass materials. Using cryogenic cooled TGG ceramics, new laser systems with both of high pulse energy and high average power such as a fusion reactor driver would be realized.

\section{References}

1) A. M. Korsunsky, J. Liu, D. Laundy, M. Golshan, and K. Kim: J. Strain Analysis 41, (2006) 113.

2) D. Ashkenasi, A. Rosenfeld, H. Varel, M. Wähmer and E. E. B. Campbell: Appl. Surf. Sci. 120, (1997) 65.

3) K. Nawata, Y. Ojima, M. Okida, T. Ogawa, and T. Omatsu: Opt. Exp. 14, (2006) 10657.

4) M. I. K. Santala, M. Zepf, F. N. Beg, E. L. Clark, A. E. Dangor, K. Krushelnick, M. Tatarakis, I. Watts, K. W. D. Ledingham, T. McCanny, et al: Appl. Phys. Lett. 78, (2001) 19.

5) J. D. Kmetec, C. L. Gordon, III, J. J. Macklin, B. E. Lemoff, G. S. Brown, and S. E. Harris: Phys. Rev. Lett. 68, (1992) 1527.

6) N. Miyanaga, H. Azechi, K.A. Tanaka, T. Kanabe, T. Jitsuno, J. Kawanaka, Y. Fujimoto, R. Kodama, H. Shiraga, K. Knodo, et al: in Inertial Fusion Sciences and Applications 2005, J.-C. Gauthier, et al. ed. (EDP sciences, Les Ulis cedex A, France, 2006), p. 81.

7) W.F. Krupke: Fusion Technol. 15, (1989) 377.

8) T. Kawashima, T. Kanabe, H. Matsui, E. Eguchi, M. Yamanaka, Y. Kato, M. Nakatsuka, Y. Izawa, S. Nakai, T.Kanzaki and H. Kan: Jpn. J. Appl. Phys. 40, (2001) 6415.

9) J. D. Mansell, J. Hennawi, E. K. Gustafson, M. M. Fejer, R. L. Byer, D. Clubley, S. Yoshida, and D. H. Reitze: Appl. Opt. 40, (2001) 366.

10) H. Seito, M. Kawase and M. Saito: Appl. Opt. 24, (1985) 2300.

11) J. Ballato and E. Snitzer: Appl. Opt. 34, (1995) 6848.

12) T. Shintaku and T. Uno: J. Appl. Phys. 76, (1994) 8155.

13) V. I. Chani, A. Yoshikawa, H. Machida, T. Satoh and T. Fukuda: Journal of Crystal Growth 210, (2000) 663.

14) J. A. Davis and R. M. Bunch: Appl. Opt. 23, (1984) 633.

15) M. Y. A. Raja, D. Allen, and W. Sisk: Appl. Phys. Lett. 67, (1995) 2123.

16) N. Grumman, TGG data sheet. (2006). A.A.Kaminskii, H.J.Eichler, P. Reiche, and R. Uecker: Laser Phys. Lett. 2, (2005) 489.

17) R. Wynands, F. Diedrich, D. Meschede, H.R. Telle, Review of Scientific Instruments 63, (1992) 5586.

18) E.A. Khazanov, N.F. Andreev, A.N. Mal'shakov, O.V. Palashov, A.K. Poteomkin, A.M. Sergeev, A.A. Shaykin, V.V. Zelenogorsky, I. Ivanov, R.S. Amin, et al: IEEE J. Quantum Electron. 40, (2004) 1500.

19) G. Mueller, R.S. Amin, D. Guagliardo, D. McFeron, R. Lundock, D.H. Reitze, D.B. Tanner: Classical and Quantum Gravity 19, (2002) 1793 
20) X. Chen, R. Galemezuk, B. Salce, B. Lavorel, C. Akir, L. Rajaonah: Solid State Communications 110, (1999) 431.

21) G. A. Slack and D. W. Oliver: Phys. Rev. B 4, (1971) 592.

22) Efim A. Khazanov: Proc. SPIE 4968, (2003) 115.

23) Mikhail A. Kagan and Efim A. Khazanov: Appl. Opt. 43, (2004) 6030.

24) T. Yanagitani, H. Yagi, and M. Ichikawa: Japanese Patent, 10-101333 (1998).

25) T. Yanagitani, H. Yagi, and M. Ichikawa: Japanese Patent, 10-101411 (1998).

26) J. Lu, H. Yagi, K. Takaichi, T. Uematsu, J.-F. Bisson, Y. Feng, A. Shirakawa, K.-I. Ueda, T. Yanagitani, and A. A. Kaminskii: Appl. Phys. B 79, (2004) 25.

27) J. Kawanaka, S. Tokita, H. Nishioka, M. Fujita, K.Yamakawa, K. Ueda and Y. Izawa: Laser Physics 15, (2005) 1306.
28) S. Tokita, J. Kawanaka, Y. Izawa, M. Fujita, and T. Kawashima: Opt. Exp. 15, (2007) 3955.

29) J. Kawanaka, H. Nishioka, N. Inoue, and K. Ueda: Appl. Opt. 40, (2001) 3542.

30) J. Kawanaka, K. Yamakawa, H. Nishioka, and K. Ueda: Opt. Lett. 28, (2003) 2121.

31) D. J. Ripin, J. R. Ochoa, R. L. Aggarwal, and T. Y. Fan: Opt. Lett. 29, (2004) 2154

32) N. P. Barnes, L. B. Petway: J. Opt. Soc. Am. B 9, (1992) 1912.

33) D.S. Zheleznov, A.V. Voitovich, I.B.Mukhin, O.V. Palashov, E.A. Khazanov: Quantum Electronics 36, (2006) 383.

34) R. Yasuhara, S. Tokita, J. Kawanaka, T. Kawashima, H. Kan, H. Yagi, H. Nozawa, T. Yanagitani, Y. Fujimoto, H. Yoshida, and M. Nakatsuka: Opt. Exp. 15, (2007) 11255. 